\title{
Stability and Plasticity of Developing Synapses in Hippocampal Neuronal Cultures
}

\author{
F. Woodward Hopf, ${ }^{1}$ Jack Waters, ${ }^{2}$ Samar Mehta, ${ }^{3}$ and Stephen J. Smith ${ }^{4}$ \\ ${ }^{1}$ Ernest Gallo Clinic and Research Center, Emeryville, California 94608, ${ }^{2}$ Abteilung Zellphysiologie, Max-Planck-Institut fuer \\ medizinische Forschung, D69120 Heidelberg, Germany, ${ }^{3}$ Group in Neuroscience, University of California San Diego, La \\ Jolla, California 92093, and ${ }^{4}$ Department of Molecular and Cellular Physiology, Stanford University Medical School, \\ Stanford, California 94305
}

To explore mechanisms governing the formation, stability, and elimination of synapses during neuronal development, we used FM 1-43 fluorescence imaging to track vesicle turnover at $>7000$ individually identified developing synapses between embryonic rat hippocampal neurons in culture. The majority of presynaptic boutons were stable in efficacy and position over a period of $1.5 \mathrm{hr}$. Activity, evoked by burst-patterned field stimulation, decreased presynaptic function across the population of boutons, an effect that required NMDA receptor activation.

Traditional neuroanatomic studies of CNS development (LeVay et al., 1980; Stryker and Harris, 1986; Katz and Shatz, 1996) combined with physiological studies of neuromuscular junction formation (Nguyen and Lichtman, 1996; Colman et al., 1997; Fitzsimonds and Poo, 1998) suggest that opposing processes of synapse formation and elimination cooperate to shape developing patterns of connectivity in neural networks. The processes of synapse formation and synapse elimination may depend on neural activity and, at least in some cases, on the activation of NMDAtype glutamate receptors (Katz and Shatz, 1996). Unfortunately, synapse formation, stability, and elimination have rarely been studied at the level of individual CNS synapses. Such information is rare because electrical methods for the study of synaptic physiology provide only very limited information about individual synapses and are difficult to apply over time scales of sufficient length to explore developmental processes. Nonetheless, several recent studies have shown that synaptic formation can occur quite rapidly (<60 min) in cultured neurons (Ahmari et al., 2000; Friedman et al., 2000).

FM 1-43 fluorescence imaging methods (Betz et al., 1992) have proved very useful for the analysis of presynaptic function at the level of individual CNS synapses (Ryan et al., 1993; Ryan and Smith, 1995; Murthy et al., 1997; Friedman et al., 2000). FM 1-43 is an amphipathic styryl dye that is taken up by recycling synaptic vesicles and can be used to quantitatively investigate presynaptic vesicle turnover. FM 1-43 methods are capable not only of isolating the function of individual boutons but of doing so with

Received June 28, 2001; revised Oct. 30, 2001; accepted Nov. 7, 2001.

This work was supported by National Institutes of Health Grant NS28587 and National Institute of Mental Health Grant MH48108 to S.J.S. We thank Cindy Adams, Susanne Ahmari, Chris Hazuka, and Jamie Jontes for critical reading of this manuscript and Alison Lam for assistance with immunocytochemistry.

Correspondence should be addressed to F. Woodward Hopf, Ernest Gallo Clinic and Research Center, 5858 Horton Street, Suite 200, Emeryville, CA 94608. E-mail: woody@egcrc.net.

Copyright (ㄷ) 2002 Society for Neuroscience $\quad 0270-6474 / 02 / 220775-07 \$ 15.00 / 0$
Decreased FM 1-43 staining correlated with low synapsin-I and synaptophysin immunoreactivities, suggesting that decreased presynaptic function was commensurate with synaptic disassembly. These observations provide new information on the stability of developing presynaptic function and suggest that NMDA receptor activation may regulate the stability of developing synapses.

Key words: hippocampus; activity; NMDA receptor; synaptogenesis; plasticity; FM 1-43 very high throughput, such that hundreds of boutons can be analyzed individually in a single experiment. Furthermore, these methods are relatively unobtrusive and can be applied repeatedly to measure function at a given synapse over substantial periods of time. We have used repeated applications of FM 1-43 to rat embryonic hippocampal cultures to study synaptic development at 7-9 d in vitro. This is a period of peak synaptogenesis immediately after the first functional synapses appear in this preparation (Fletcher et al., 1991). Here, we have used persistent burst firing to test the role of neuronal activity and NMDA receptors in possible activity-dependent changes in synaptic function, measured with FM 1-43, and presynaptic protein levels, measured with retrospective quantitative immunohistochemistry.

\section{MATERIALS AND METHODS}

FM 1-43 labeling and fluorescence imaging. Primary dissociated cultures were prepared from the embryonic hippocampus of Sprague Dawley rats according to the methods of Banker and Goslin (1996). A coverslip containing neurons was placed in a low-volume $(\sim 60 \mu \mathrm{l})$ laminar perfusion chamber and mounted on the stage of a confocal laser scanning microscope at $34^{\circ} \mathrm{C}$ (Adams et al., 1996). Cells were continuously perfused with a modified Tyrode's solution of the following composition (in mM): 119 $\mathrm{NaCl}, 2.5 \mathrm{KCl}, 2 \mathrm{CaCl}_{2}, 2 \mathrm{MgCl}_{2}, 25$ HEPES, pH 7.4, and 30 glucose. Extracellular solution also contained $50 \mu \mathrm{M}$ AP-5 (D,L-2-amino-5phosphonovaleric acid) and $10 \mu \mathrm{M}$ 6-cyano-7-nitroquinoxaline-2,3-dione (CNQX) during FM 1-43 measurements to reduce spontaneous activity. During the "intertest period interval," neurons were perfused with Tyrode's solution in the absence of any drugs, unless otherwise specified.

An FM 1-43 measurement performed on the microscope stage began with the addition of $15 \mu \mathrm{M}$ FM 1-43 (Molecular Probes, Eugene, OR) to the perfusing solution. One hundred stimuli were delivered electrically at $10 \mathrm{~Hz}$ (platinum field electrodes, $1-\mathrm{msec}$ stimuli, $50 \mathrm{~V} / \mathrm{cm}$ ), causing synaptic exocytosis. FM 1-43 was removed from the perfusion chamber $20 \mathrm{sec}$ after stimulation was terminated, allowing time for FM 1-43 uptake by endocytosis (Ryan and Smith, 1995). Cells were washed for 10 min, and three images were acquired. Cells were then exposed to 600 stimuli at $10 \mathrm{~Hz}$ to release endocytosed dye, and another image was acquired. Six hundred stimuli were sufficient to release all endocytosed FM 1-43 (data not shown). An automated, motorized stage was used to gather data at multiple sites in a single coverslip (Adams et al., 1996). 
The FM 1-43-associated fluorescence for a given synapse $(\Delta \mathrm{F})$ was calculated by subtracting the fluorescence intensity in the unloaded frame (representing nonspecific staining) from the averaged intensity of the first two loaded frames. Fluorescence intensities after a 100 -stimulus loading protocol correlated with intensities measured after a 600stimulus protocol (data not shown). Therefore, FM 1-43 staining after 100 stimuli most likely reflects the size of the total vesicle pool.

Each image was constructed from a 2-plane z-stack (1 $\mu \mathrm{m}$ interval) collected with the confocal pinhole in the open position (resulting in a depth of field of 5-10 $\mu \mathrm{m}$ ). These measures greatly reduce possible errors associated with slight changes in plane of focus; preliminary experiments indicated that this approach offered an accurate measurement of fluorescence intensity with minimal light exposure. The intensities of fluorescent puncta were determined offline. Average fluorescence intensity was calculated for a region of interest containing a single punctum. The dimensions of each region of interest was 5 pixels by 5 pixels $(0.9 \times 0.9$ $\mu \mathrm{m})$. Regions of interest were initially positioned by eye and then corrected for the center of mass of each punctum by an automated procedure in each successive frame. New puncta were identified by eye, and a mask was placed visually for each such punctum. $\Delta \mathrm{F} 1$ data from each coverslip were normalized to a mean of 66 intensity units before data from different sites were pooled. Data were normalized to correct for possible changes in laser power and gain between experiments. For analyses, the investigator was blind to the experimental condition.

All reagents except FM 1-43 were purchased from Sigma/RBI (St. Louis, MO).

Retrospective immunocytochemistry. At the end of the viewing period, some cells were processed for immunocytochemistry as described previously (Ziv and Smith, 1996). Cells were fixed with formaldehyde 5 min after $\Delta \mathrm{F} 2$ had been determined. After fixation and blocking, cells were incubated overnight with an affinity-purified rabbit anti-synapsin-Ia/b polyclonal antibody $(1: 1000)$ at $4^{\circ} \mathrm{C}$. After washing, cells were incubated for $1 \mathrm{hr}$ at room temperature with fluorescein isothiocyanate anti-rabbit IgG (Jackson ImmunoResearch, West Grove, PA). Synapsin-I and synaptophysin (1:100) immunoreactivity were normalized to a mean of 75 intensity units (after background subtraction of $\sim 15$ intensity units). Antibodies were purchased from Chemicon International, Inc. (Temecula, CA).

\section{RESULTS}

\section{Measurement of synaptic vesicle turnover at individual presynaptic sites}

Dissociated cultures were prepared from embryonic rat hippocampi using the methods of Banker and Goslin (1996). We measured presynaptic vesicle turnover at visually distinct puncta using established FM 1-43 imaging methods (Ryan et al., 1996). Platinum field electrodes were used to evoke action-potential firing to load and unload FM 1-43. In all experiments, dye loading was induced by 100 stimuli with dye in the extracellular solution. Cells were then washed to remove extracellular dye, and images were acquired. FM 1-43 was released with 600 stimuli, and images of background (nonreleasable) staining were acquired.

After FM 1-43 loading, we observed large numbers of fluorescent puncta. A typical example is given in Figure $1 A$. Presynaptic function at each punctum was measured as a difference between fluorescence intensities $(\Delta \mathrm{F})$ in the loaded (Fig. $1 A, 1,2)$ and unloaded (data not shown) conditions (i.e., before and after the 600 -stimulus unloading train). To examine changes in presynaptic function over time, we conducted two FM 1-43 loading and unloading cycles (called "FM 1-43 test periods") separated by 1.5 hr. A comparison of images taken during the two test periods is shown in Figure $1 A, 3$. Although the puncta are not perfectly aligned after $90 \mathrm{~min}$, the majority of puncta can be clearly identified at both time points.

In the course of our experiments, we observed a population of mobile FM 1-43-loaded puncta. These puncta moved at a rate of $1.96 \pm 0.25 \mu \mathrm{m} / \mathrm{min}$ (determined for 18 puncta), consistent with recent reports that describe mobile, nonsynaptic clusters of vesi-
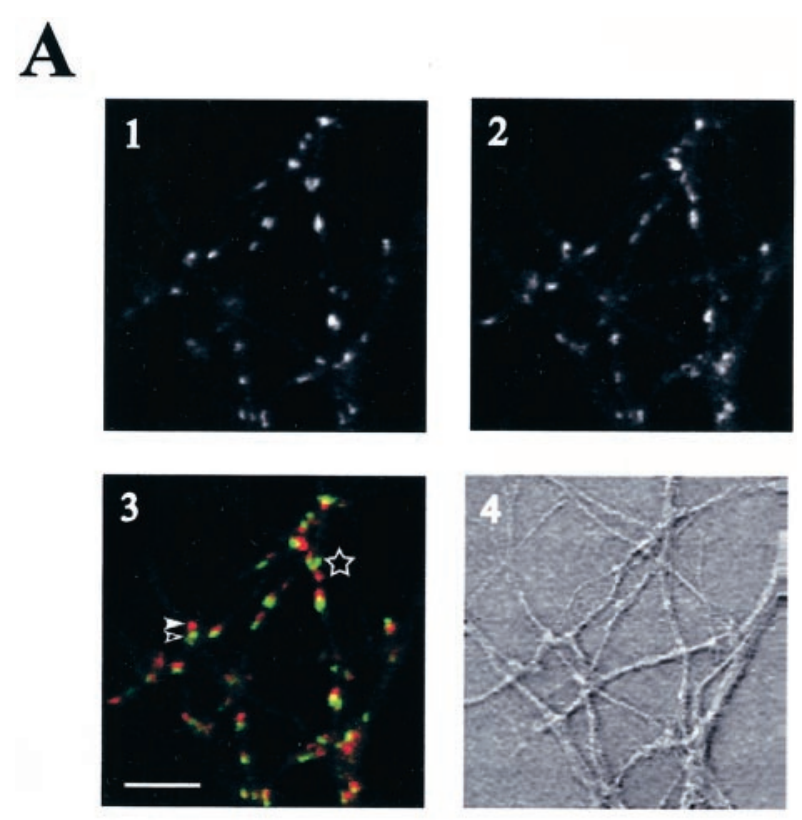

B

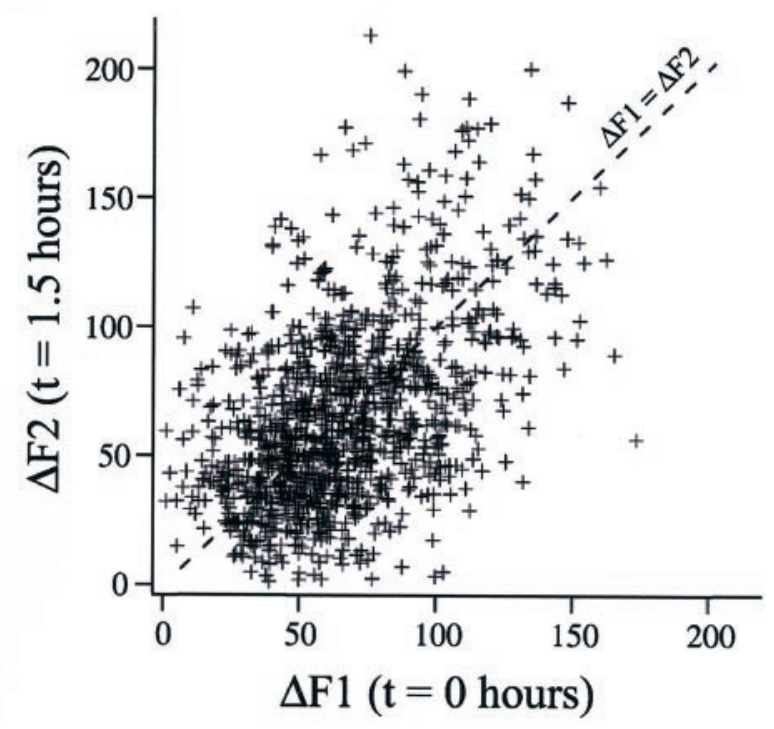

Figure 1. Changes in presynaptic function across $1.5 \mathrm{hr} . A$, Images of FM $1-43$ loading at the first $(A 1$, green in $A 3)$ and second $(A 2$, red in $A 3)$ test period. Scale bar, $10 \mu \mathrm{m}$. Despite small apparent motions of puncta between the first (open arrowhead) and second (closed arrowhead) test periods, the majority of puncta can be clearly identified at both time points. The region to the left of the star indicates an area in which punctal identity could not be determined with certainty; consequently, this area was excluded from analysis. $B$, An example of FM 1-43 loading in a population of defined puncta during the first and second test periods ( $\Delta \mathrm{F} 1$ and $\Delta \mathrm{F} 2$, respectively). These data correspond to cells subjected to stimulation in the presence of AP-5. The dashed line indicates where $\Delta \mathrm{F} 1=\Delta \mathrm{F} 2$.

cles that load and unload FM 1-43 during stimulation (Kraszewski et al., 1995; Dai and Peng, 1996). To assess changes in presynaptic function at a given punctum, we needed to conclusively identify the position of that punctum through time. Therefore, in every experiment we assayed for mobility of puncta by acquiring three images at 3 min intervals before unloading. Only 
puncta that were in the same position across all three frames ("immobile" puncta) were included for additional analysis. There were no differences in destaining kinetics between positionally stable and mobile puncta (data not shown), consistent with results from Dai and Peng (1996).

To assay for slower movements of immobile puncta across hours, neurons were loaded with FM 1-43 and imaged every 10 min for $1.5 \mathrm{hr}$. Only 40 of 951 FM 1-43 puncta (4.2\%) exhibited movements $>1 \mu \mathrm{m}$ during any $10 \mathrm{~min}$ period. These 40 puncta were positionally stable for most of the recording period but moved at a rate of $0.15 \pm 0.03 \mu \mathrm{m} / \mathrm{min}$ for an interval lasting $20.9 \pm 2.7 \mathrm{~min}$. Such levels of motions $(\sim 3.1 \mu \mathrm{m}$ total distance traveled in the 90 min period) were small compared with the mean distance between nearest neighbor puncta $(4.56 \pm 0.12 \mu \mathrm{m}$, determined for 494 puncta). Therefore, our methods ensured that only positionally stable puncta were selected for additional analysis.

\section{NMDA receptor activity promotes loss of presynaptic function}

Figure $1 B$ shows examples of FM 1-43 fluorescence intensities in a population of defined puncta during the first and second test periods ( $\Delta \mathrm{F} 1$ and $\Delta \mathrm{F} 2$, respectively). In the majority of puncta, FM 1-43 signals in the first and second test periods were similar. Puncta exhibiting a pronounced change in presynaptic function were relatively uncommon (see also Fig. $2 B$ ). Therefore, to analyze these populations, we examined thousands of individually identified boutons.

We measured changes in presynaptic FM 1-43 fluorescence at each synaptic bouton and compared these paired values across large populations of puncta to identify conditions under which changes in presynaptic function occurred. Under resting (control) conditions, presynaptic function exhibited a small decrease, with $\Delta \mathrm{F} 2$ being significantly smaller than $\Delta \mathrm{F} 1(n=401$ puncta; $p=$ 0.0048; Wilcoxon signed rank-sum test). The presence of AP-5 and CNQX during the interstimulus interval $(n=1321)$ did not significantly reduce the shift in presynaptic function $(p=0.61$; Mann-Whitney rank-sum test) observed in the absence of stimulation. To test for the effects of activity on synaptic function, neurons were stimulated to fire action potentials during the $1.5 \mathrm{hr}$ interval between FM 1-43 test periods (called the intertest period interval). Stimuli were delivered in $3 \mathrm{sec}$ bursts at $20 \mathrm{~Hz}$, repeated every $60 \mathrm{sec}$ for $75 \mathrm{~min}$. As in controls, stimulated preparations exhibited a significant decrease in presynaptic function $(n=3438$ puncta; $p<0.0001$; Wilcoxon signed rank-sum test). In contrast, when the preparation was perfused with either $500 \mathrm{~nm}$ tetrodotoxin (TTX) or $50 \mu \mathrm{M}$ AP-5 during the intertest period interval, no significant decrease in presynaptic activity was observed after stimulation ( $n=1082, p=0.135$ and $n=1315, p=0.05$ for TTX and AP-5, respectively; Wilcoxon signed rank-sum tests).

These data suggest that electrical stimulation may act through NMDA receptors to alter presynaptic function. To allow comparison among different treatments, changes in presynaptic function were expressed as ratios of FM 1-43 fluorescence intensities during the first and second test periods $(\Delta \mathrm{F} 2 / \Delta \mathrm{F} 1$ ratio $)$. Ratios of $<1$ indicate a loss of presynaptic function, whereas ratios of $>1$ indicate a gain. The median $\Delta \mathrm{F} 2 / \Delta \mathrm{F} 1$ ratio for each condition is plotted in Figure $2 A$, along with error bars indicating the scatter of the data in each population (see Fig. $2 A$ legend).

$\Delta \mathrm{F} 2 / \Delta \mathrm{F} 1$ ratios of synapses in the different treatment groups were compared using the Kruskal-Wallis test (nonparametric counterpart of an ANOVA). This revealed a significant difference

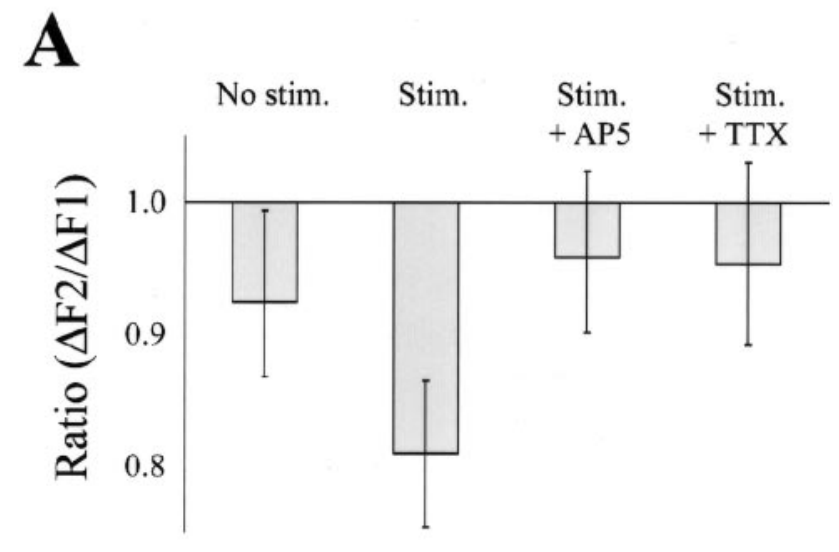

B

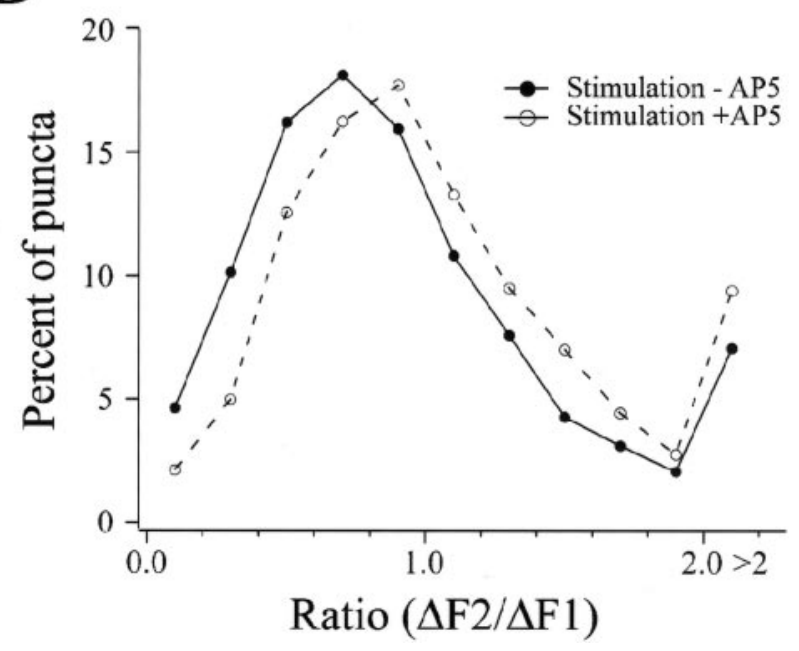

Figure 2. Burst stimulation resulted in a decrease in presynaptic function. $A$, Median change in presynaptic function under indicated conditions. Error bars indicate the values for data points removed from the median by $5 \%$ of the population size. Median values were as follows: controls, $0.93(n=401)$; stimulated, $0.81(n=3438)$; stimulated in AP-5, $0.96(n=1082)$; stimulated in TTX, $0.95(n=1315) . B$, Proportion of puncta exhibiting a given $\Delta \mathrm{F} 2 / \Delta \mathrm{F} 1$ ratio in cells subjected to burst stimulation in the presence (open symbols) and absence ( filled symbols) of 50 $\mu \mathrm{M}$ AP-5. The data are displayed as a frequency histogram using 0.2 ratio-unit bins.

between the groups $(p<0.0001)$. Comparisons were then made between groups using the Mann-Whitney rank-sum test. Data from preparations exposed to evoked stimulation during the intertest period interval showed a significantly greater decrease in presynaptic function (smaller $\Delta \mathrm{F} 2 / \Delta \mathrm{F} 1$ ratios) than controls ( $p<$ 0.0001; Mann-Whitney rank-sum test). Preparations stimulated in the presence of AP-5 or TTX during intertest periods exhibited smaller decreases in function than preparations stimulated in the absence of drugs (both $p<0.0001$; Mann-Whitney rank-sum tests). Furthermore, there was no significant difference between unstimulated neurons and neurons receiving intertest period stimulation in the presence of AP-5 or TTX $(p>0.25$ for each; Mann-Whitney rank-sum tests). These additional analyses clearly indicate that stimulation caused a decrease in presynaptic function through activation of NMDA receptors.

Changes in $\Delta \mathrm{F} 2 / \Delta \mathrm{F} 1$ across the population of puncta were examined using frequency histograms. A leftward shift in distri- 
bution of $\Delta \mathrm{F} 2 / \Delta \mathrm{F} 1$ ratios was evident after stimulation without AP-5 (Fig. $2 B)(p<0.01$; Kruskal-Wallis rank-sum test). These data suggest that activity led to a small weakening of function across a large population of synapses rather than to a large effect restricted to a few puncta (see also Fig. 4).

Finally, we examined whether stimulation at $1 \mathrm{~Hz}$ during the entire intertest period would lead to changes in synaptic function similar to those with higher-frequency stimulation. After $1 \mathrm{~Hz}$ intertest period stimulation, $\Delta \mathrm{F} 2$ was significantly different from $\Delta \mathrm{F} 1$ ( $n=1300 ; p<0.0001$; Wilcoxon signed rank test). However, $1 \mathrm{~Hz}$ stimulation did not produce $\Delta \mathrm{F} 2 / \Delta \mathrm{F} 1$ ratios that were significantly different from unstimulated controls ( $p=0.99$; Man$\mathrm{n}$-Whitney rank-sum test). Furthermore, $\Delta \mathrm{F} 2 / \Delta \mathrm{F} 1$ ratios after 1 $\mathrm{Hz}$ stimulation were significantly lower than those observed with higher-frequency stimulation $(p<0.0001$; Mann-Whitney ranksum test). Thus, $1 \mathrm{~Hz}$ stimulation did not result in a change in $\Delta \mathrm{F} 2 / \Delta \mathrm{F} 1$ that was different from unstimulated control neurons, although the number of stimuli delivered to cells by $1 \mathrm{~Hz}$ and higher-frequency stimulation was similar (60 stimuli per sec).

\section{Loss of presynaptic function was correlated with lower synapsin-I and synaptophysin immunoreactivity}

To investigate whether the observed functional changes were accompanied by changes in presynaptic structure, neurons were fixed after the second FM 1-43 test period, and the levels of the presynaptic proteins synapsin-I and synaptophysin were examined using immunocytochemistry. Figure $3 A$ shows examples of fluorescence images of FM 1-43 (Figs. $3 A, 1,2$ ) and synapsin-I immunoreactivity (Fig. 3A, 3). Puncta with robust presynaptic function during the second FM 1-43 test period were brightly labeled with antibodies to synapsin-I (Fig. $3 A$, arrowheads). In contrast, puncta with low presynaptic function during the second loading period $(\Delta \mathrm{F} 2)$ also exhibited lower synapsin-I immunoreactivity (Fig. $3 A$, arrows). Hence, presynaptic function immediately before fixation $(\Delta \mathrm{F} 2)$ and synapsin-I immunoreactivity were highly correlated (Fig. 3B), as reported previously (Sporns and Jenkinson, 1997; Staple et al., 1997).

Figure $4 A$ shows the relationship between changes in presynaptic function $(\Delta \mathrm{F} 2 / \Delta \mathrm{F} 1$ ratio $)$ and synapsin-I immunoreactivity for synapses subjected to stimulation in the presence $(n=1341$ puncta) and absence ( $n=884$ puncta) of AP-5. Synapsin-I immunoreactivity was reduced by a substantial and significant extent in puncta exhibiting decreased presynaptic function (a $\Delta \mathrm{F} 2 / \Delta \mathrm{F} 1$ ratio of $<1$ ) relative to puncta exhibiting no change in presynaptic function (a $\Delta \mathrm{F} 2 / \Delta \mathrm{F} 1$ ratio of 1 ; Fig. $4 A$ ). This relationship between synapsin-I immunoreactivity and $\Delta \mathrm{F} 2 / \Delta \mathrm{F} 1$ ratio was observed both in the presence and absence of AP-5.

Like synapsin-I, the synaptic vesicle membrane protein synaptophysin also exhibited a significantly lower immunoreactivity in puncta with a low $\Delta \mathrm{F} 2 / \Delta \mathrm{F} 1$ ratio $(n=497$ puncta; Fig. $4 B)$. Immunoreactivities for synapsin and synaptophysin were correlated (data not shown).

The relationships between $\Delta \mathrm{F} 2 / \Delta \mathrm{F} 1$ ratio and synaptic protein immunoreactivities were not the result of differences in initial presynaptic function, because $\Delta \mathrm{F} 1$ levels were not different between puncta with reduced presynaptic function and those for which presynaptic function did not change (Fig. 4C). For puncta with increased presynaptic function, $\Delta \mathrm{F} 1$ of course had a smaller value than $\Delta \mathrm{F} 2$, and puncta with the highest ratios therefore had the lowest $\Delta \mathrm{F} 1$ values. This trend is clearly visible in Figure $4 C$ and probably reflects a ceiling effect attributable to the finite number of vesicles in each presynaptic bouton (Ryan et al., 1997;
A
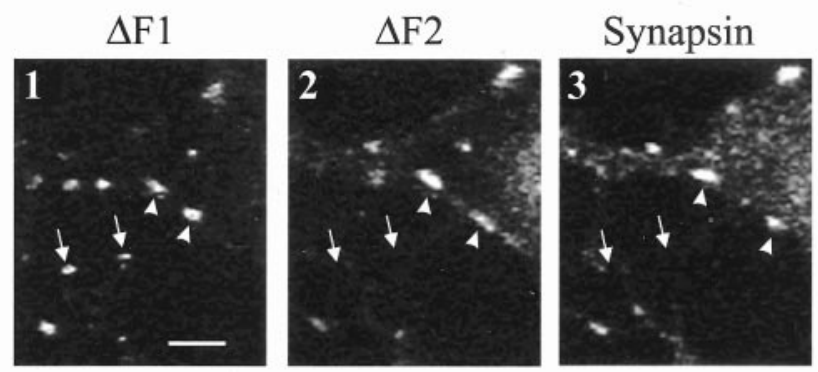

B

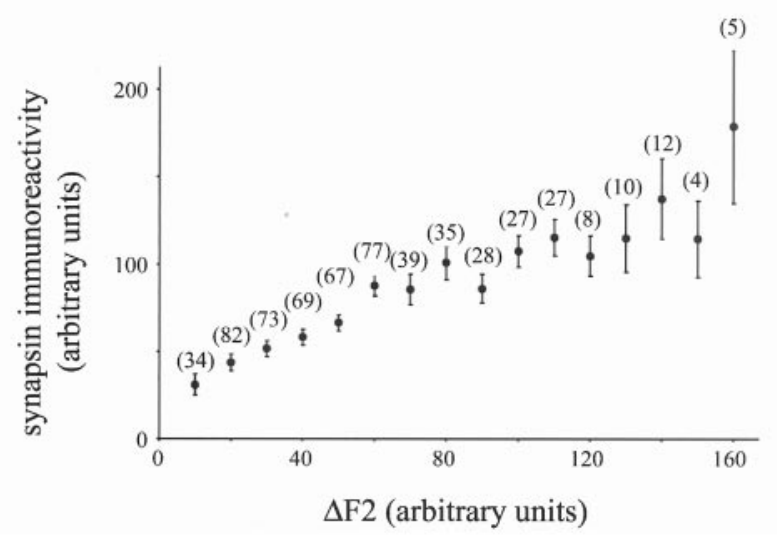

Figure 3. Images showing an example of $\Delta \mathrm{F} 1, \Delta \mathrm{F} 2$, and synapsin-I immunoreactivity. $A$, Puncta exhibiting presynaptic function in the first test period $(\Delta \mathrm{F} 1)$ but not in the second test period $(\Delta \mathrm{F} 2)$ did not exhibit synapsin-I staining (arrows). Puncta that exhibited robust FM 1-43 uptake in the first and second test periods showed synapsin-I staining (arrowheads). Scale bar, $5 \mu \mathrm{m}$. B, Presynaptic function in the second test period $(\Delta \mathrm{F} 2)$ correlated with levels of synapsin-I. The numbers of puncta from which points are derived are given in parentheses.

Schikorski and Stevens, 1997). This ceiling effect also contributed to the relationship between $\Delta \mathrm{F} 2 / \Delta \mathrm{F} 1$ and synapsin-I immunoreactivity (described above). Because of this ceiling, neurons with a low $\Delta \mathrm{F} 2 / \Delta \mathrm{F} 1$ value could have only lower values of $\Delta \mathrm{F} 2$, whereas neurons with a $\Delta \mathrm{F} 2 / \Delta \mathrm{F} 1$ ratio of 1 could have a wider range of $\Delta \mathrm{F} 2$ values, with a higher average value of $\Delta \mathrm{F} 2$. Thus, given the correlation of $\Delta \mathrm{F} 2$ and synapsin-I (see above), a relationship was also observed between $\Delta \mathrm{F} 2 / \Delta \mathrm{F} 1$ and synapsin-I levels.

\section{Direct imaging of vesicle removal}

Synaptic elimination probably requires the removal of vesicles, either individually or as clusters. We have observed a few examples of such events. Figure $5 A$, site 1 , illustrates movement of vesicles from an identified punctum (indicated by an arrow). Some of these vesicles remained in the adjacent axonal branch as diffuse FM 1-43 staining. The pattern of FM 1-43 uptake in the second test period was very similar to the FM 1-43 fluorescence observed just before the first unloading stimulus train (see frame at $90 \mathrm{~min}$ ), suggesting that the observed movement of FM 1-43 fluorescence represents movement of functional vesicles away from the initial release site. Figure $5 A$, site 2 , demonstrates the division of a punctum, where the sum of the fluorescence intensities of the two daughter puncta equals that of the parent punctum (illustrated in Fig. $5 B$ ), indicating that the total number of FM 1-43-loaded vesicles remained constant. Note that daughter 

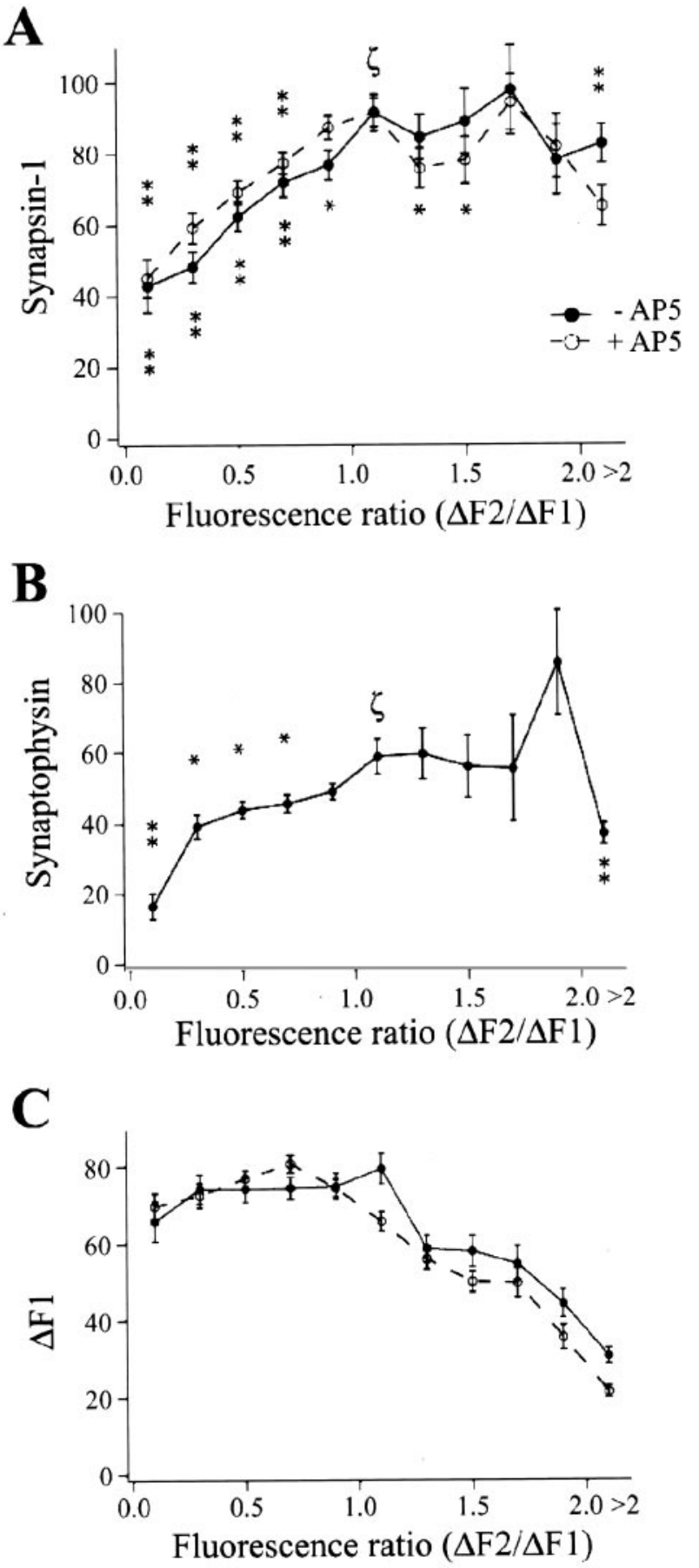

Figure 4. Decreased presynaptic function was accompanied by lower levels of synapsin-I and synaptophysin in individual boutons. $A$, Boutons exhibiting decreased presynaptic function (indicated by a $\Delta \mathrm{F} 2 / \Delta \mathrm{F} 1$ ratio of $<1.0$ ) showed decreased levels of synapsin-I relative to puncta exhibiting no change in presynaptic function $(a \mathrm{~F} 2 / \Delta \mathrm{F} 1$ ratio of $\sim 1)$. This pattern was observed regardless of whether AP-5 was present or absent during bursting stimulation. $B$, Boutons exhibiting decreased presynaptic function showed decreased levels of synaptophysin. Data are binned by 0.2 ratio units, except for puncta with a $\Delta \mathrm{F} 2 / \Delta \mathrm{F} 1$ ratio of $>2$, which are grouped. $p<0.01$ (stacked asterisks) and $p<0.05$ (single asterisk) for data points significantly different from the point labeled with $\zeta$ (Mann-Whitney rank-sum test). Significance values were not calculated for $\Delta \mathrm{F} 1$ data for a ratio of $>1.2$. $C$, Boutons exhibiting decreased presynaptic function had the same initial FM 1-43 loading as boutons exhibiting no change in presynaptic function.
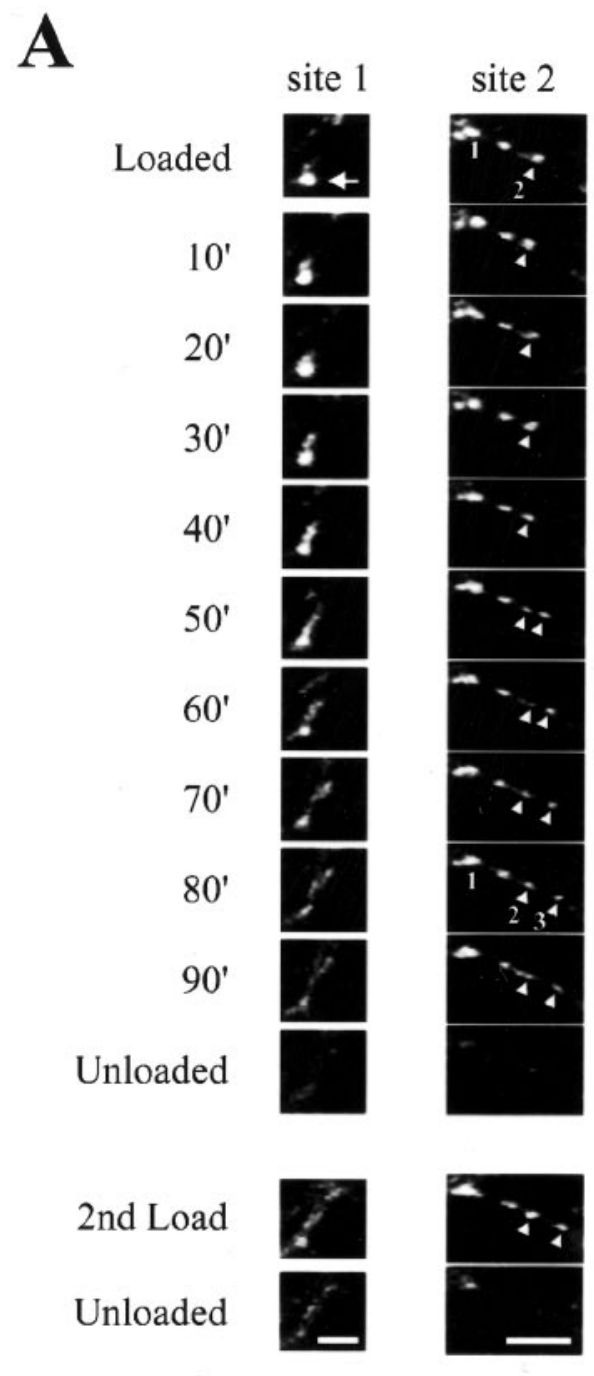

B

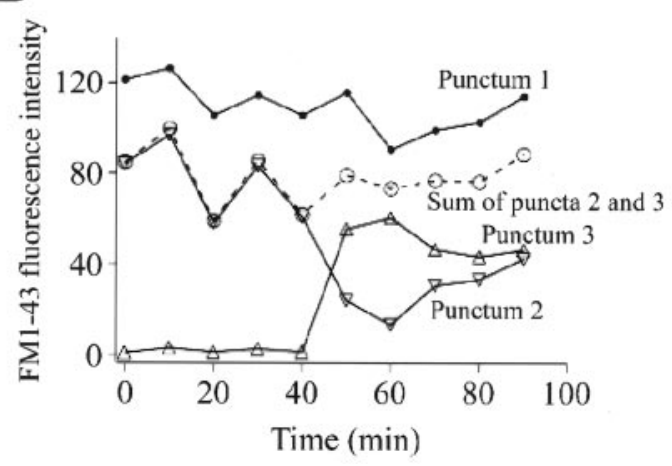

Figure 5. Examples of the removal of vesicles from synapses. Boutons were loaded with FM 1-43 and imaged every $10 \mathrm{~min}$. A, site 1, An example of trafficking of vesicles from a bright punctum (arrow). A, site 2, An example of the division of a punctum (arrowheads). B, Quantification of changes in the fluorescence intensity of puncta in site 2. The sum of intensities from puncta 2 and 3 remained constant, indicating no net loss of vesicles. Puncta exhibited minimal photobleaching (see punctum 1). Scale bars, $5 \mu \mathrm{m}$.

puncta maintained their levels of presynaptic function, having appropriate $\Delta \mathrm{F}$ values during the second FM 1-43 test period (Fig. 5B). 


\section{DISCUSSION}

\section{Activity and the magnitude of synaptic suppression}

We used serially repeated FM 1-43 tests to investigate changes in presynaptic function at nascent synapses in embryonic hippocampal neurons. We observed a trend toward decreased presynaptic function after stimulation, which was inhibited by AP-5 and by TTX. Using hippocampal cultures from newborn rats, Lissin et al. (1998) observed an AP-5- and TTX-sensitive decrease in staining for glutamate receptors after increased excitatory activity, suggesting that NMDA receptor activation results in decreased postsynaptic receptor clustering in postnatal hippocampal cultures. In embryonic hippocampal cultures, inhibition of spontaneous activity with AP-5 or TTX results in an increase in NMDA receptor clustering (Rao and Craig, 1997). These studies of postsynaptic receptor clustering in hippocampal cultures illustrate the role of NMDA receptor activity in limiting postsynaptic receptor density. Our data complement these previous findings, adding a presynaptic correlate. Together, these data suggest that NMDA receptor activity plays an important role in controlling both presynaptic and postsynaptic development in the embryonic and neonatal hippocampus.

Neuronal activity was not directly monitored in our experiments. It is therefore possible that AP-5 influenced presynaptic function by directly reducing neuronal activity or instead by decreasing overall network activity through actions on the NMDA receptor. Regardless of the mechanism of action of AP-5, however, signaling through the NMDA receptor clearly plays a significant role in activity-dependent phenomena. Also, the stimulations used for FM 1-43 loading and unloading might themselves influence activity-dependent phenomena. Any such effects should influence all experimental conditions and thus do not alter our conclusion that higher-frequency stimulation during the intertest period resulted in an NMDA receptor-dependent shift toward decreased presynaptic function.

One notable difference between the effects of activity on postsynaptic receptor expression (Rao and Craig, 1997) and on presynaptic function (studied here) is the magnitude of the effect of AP-5. Rao and Craig (1997) observed a 380\% increase in receptor clustering. In contrast, our principal observation was one of synaptic stability; under resting conditions, the majority of puncta $(\sim 75 \%)$ exhibited no significant change in synaptic function, and after stimulation, only a small (16\%) shift in mean presynaptic function was observed (Fig. 2). This may reflect the very different time scales of the two studies, because Rao and Craig (1997) treated cultures with AP-5 for 1-40 d, 1-2 orders of magnitude longer than we used on the microscope stage. Also, our experiments were done in the absence of growth factors, which might be required for synaptic growth. This might contribute to the relative paucity of boutons with increased presynaptic function.

We observed a large variability in FM 1-43 fluorescence measurements between the first and second test periods (Figs. $1 B$, $2 B)$. This might reflect several factors, especially the stochastic nature of vesicle release and measurement noise, in addition to actual changes in presynaptic function. Regardless of these sources of variability in the distribution of $\Delta \mathrm{F} 2 / \Delta \mathrm{F} 1$, however, we observed consistent changes in presynaptic function in relation to activity and NMDA receptor function.

Studies at the neuromuscular junction, where activity-dependent weakening of synaptic function precedes synapse elimination, suggest that loss of a synapse is a protracted affair, requiring $\geq 24 \mathrm{hr}$ for withdrawal of the presynaptic terminal (Colman et al., 1997).
Our data address the loss of function rather than loss of physical contact between presynaptic and postsynaptic elements. The fact that function may be lost in a relatively brief interval $(1.5 \mathrm{hr})$, however, may reflect a difference in the time course of comparable processes at the neuromuscular junction and in the CNS. Also, several studies using cultured hippocampal neurons to examine synaptic formation have found that recruitment of synaptic components can occur rapidly (in $<1$ hr) (Ahmari et al., 2000; Friedman et al., 2000). Thus, the dynamics of formation and elimination of CNS synapses may be much more rapid than at the neuromuscular junction. In addition, our results might suggest that elimination of CNS synapses can occur via removal of individual synapses without gross withdrawal of entire axons. However, Culican et al. (1998) have observed that different molecular components of the neuromuscular junction are eliminated at different rates, raising the possibility that our observations represent an early step in CNS synapse elimination, which ultimately results in a greater degree of axonal retraction.

Our imposed pattern of stimulation $(20 \mathrm{~Hz}$ for $3 \mathrm{sec}$ every minute for $1.5 \mathrm{hr}$ ) is similar to those associated with potentiation in the hippocampal slice (Bliss and Collingridge, 1993). Also, $1 \mathrm{~Hz}$ stimulation, which is associated with long-term depression in many studies (Bear and Abraham, 1996), did not lead to changes in presynaptic function. We are not presuming or ascribing any specific relationship between the activity-dependent phenomenon observed here and long-term potentiation or long-term depression. In this regard, the outcome of activity-dependent plasticity is not determined by stimulation frequency alone. The effect of stimulation has been shown to be dependent on the precise timing of synaptic inputs (Markram et al., 1997; Bi and Poo, 1998, 1999). Our method of stimulation should have resulted in almost simultaneous action-potential initiation throughout the population of neurons. However, we would expect the complex morphology of hippocampal neurons to result in different timing of the arrival of presynaptic and postsynaptic inputs at different synapses. Therefore, the effect on the population as a whole might be closely related to cell morphology and to the age of the neurons studied.

Ryan et al. (1996) examined presynaptic function using multiple loading of FM 1-43 but observed an increase in presynaptic function. There are several possible explanations for this difference, including the preparation of cultures from older (postnatal) animals, the different age of cultures used (3-4 weeks in culture vs 7-9 d here), and a different stimulation protocol.

FM 1-43 has also been used to examine the effect of activation of the protein kinase A system on presynaptic function. Ma et al. (1999) used a methodology similar to that in the present study, comparing presynaptic function across two FM 1-43 test periods separated by 2 hr. Ma et al. (1999) stimulated vesicle turnover using high extracellular potassium instead of electrical stimulation and were unable to identify individual boutons across the two FM 1-43 test periods. Brief application of the protein kinase A activator $\mathrm{Sp}$-cAMPs greatly increased the number of active boutons without a change in mean FM 1-43 staining. In contrast, in our study we observed a mean decrease in FM 1-43 staining with activity but a very small change in the number of active boutons. In both studies, changes in synaptic function required activity and NMDA receptor activation.

\section{Functional suppression or synaptic disassembly?}

Our methods of measuring levels of the synaptic proteins synapsin-I and synaptophysin required that the cells be fixed for immunocytochemical processing, preventing determination of 
the levels of synapsin or synaptophysin before vital staining with FM 1-43. Using this method, we cannot compare initial protein levels at "functionally stable" puncta and at puncta that displayed a loss of presynaptic function. Therefore, there are two viable models that describe our data: (1) low levels of synaptic proteins (for a given level of functional activity) make a synapse particularly susceptible to synaptic suppression, or (2) activitydependent functional suppression results in a subsequent loss of synaptic proteins. Although we cannot definitively discriminate between these possibilities, the latter would seem more likely, because loss of presynaptic function correlates with loss of immunoreactivities to both synaptophysin, a vesicle membrane protein, and synapsin-I, a protein associated with both synaptic vesicles and the cytoskeletal matrix (Valtorta et al., 1992). It seems unlikely that susceptibility to suppression is a function of both proteins. Therefore, our data suggest that functional suppression leads to an alteration in presynaptic structure consisting, at least in part, of the removal of presynaptic proteins.

We should note that 100 stimuli are insufficient to load the entire recycling pool, raising the possibility that the observed changes in presynaptic function might reflect modification of the readily releasable pool rather than the entire recycling pool. Although we cannot rule out alterations in the number of readily releasable vesicles, preliminary experiments found that 100 stimuli stained $\sim 50 \%$ of the recycling vesicle pool (determined with a 600 action-potential stimulus), which is greater than the size of the readily releasable pool (J. Waters and S. J. Smith, unpublished observations). Thus, we consider it more likely that observed activity-dependent alterations in synaptic function reflect changes in the total number of recycling vesicles.

Imaging the movement of FM 1-43-labeled vesicles from a synapse proved technically demanding, because such movements were rare. However, we have observed this phenomenon on several occasions (Fig. $5 A$ ) and have demonstrated that the resulting loss of function is quantitatively equivalent to fluorescence of the trafficked vesicles. This observation directly demonstrates that removal of presynaptic elements (in this case recycling vesicles) occurs at some synapses, and that this results in a decrease in presynaptic function.

Together, the observation of vesicle movement and the relationship between presynaptic function and the levels of presynaptic proteins suggest that one mechanism by which synaptic strength, and perhaps synaptic connectivity, is modified in the developing CNS is the removal of presynaptic structural elements, including vesicles and associated proteins. As at the postsynaptic specialization, these alterations are strongly influenced by NMDA receptor activity. The data presented here add a presynaptic correlate to the established role of NMDA receptors in the alteration of postsynaptic properties at the level of the individual CNS synapse. We should note that the nature of the signaling mechanism that is initiated by NMDA receptor activity and results in synaptic disassembly is still an open question. There are several potential candidates that might intervene in the disassembly process, including diff usable factors and growth factors (Lichtman and Colman, 2000); additional experiments are required to elucidate such mechanisms.

\section{REFERENCES}

Adams CL, Nelson WJ, Smith SJ (1996) Quantitative analysis of cadherin-catenin-actin reorganization during development of cell-cell adhesion. J Cell Biol 135:1899-1911.

Ahmari SE, Buchanan J, Smith SJ (2000) Assembly of presynaptic active zones from cytoplasmic transport packets. Nat Neurosci 3:445-451.
Banker G, Goslin K (1996) Rat hippocampal neurons in low-density culture. In: Culturing nerve cells (Banker G, Goslin K, eds), pp 251278. Cambridge, MA: MIT.

Bear MF, Abraham WC (1996) Long-term depression in the hippocampus. Annu Rev Neurosci 19:437-462.

Betz WJ, Mao F, Bewick GS (1992) Activity-dependent fluorescent staining and destaining of living motor nerve terminals. J Neurosci 12:363-375.

Bi GQ, Poo MM (1998) Synaptic modifications in cultured hippocampal neurons: dependence on spike timing, synaptic strength, and postsynaptic cell type. J Neurosci 18:10464-10472.

Bi GQ, Poo MM (1999) Distributed synaptic modification in neural networks induced by patterned stimulation. Nature 401:792-796.

Bliss TVP, Collingridge GL (1993) A synaptic model of memory: longterm potentiation in the hippocampus. Nature 361:31-39.

Colman H, Nabekura J, Lichtman JW (1997) Alterations in synaptic strength preceding axon withdrawal. Science 275:356-361.

Culican SM, Nelson CC, Lichtman JW (1998) Axon withdrawal during synapse elimination at the neuromuscular junction is accompanied by disassembly of the postsynaptic specialization and withdrawal of Schwann cell processes. J Neurosci 18:4953-4965.

Dai Z, Peng HB (1996) Dynamics of synaptic vesicles in cultured spinal cord neurons in relationship to synaptogenesis. Mol Cell Neurosci 7:443-452.

Fitzsimonds R, Poo MM (1998) Retrograde signaling in the development and modification of synapses. Physiol Rev 78:143-170.

Fletcher TL, Cameron P, De Camilli P, Banker G (1991) The distribution of synapsin I and synaptophysin in hippocampal neurons developing in culture. J Neurosci 11:1617-1626.

Friedman HV, Bresler T, Garner CC, Ziv NE (2000) Assembly of new individual excitatory synapses: time course and temporal order of synaptic molecule recruitment. Neuron 27:57-69.

Katz LC, Shatz CJ (1996) Synaptic activity and the construction of cortical circuits. Science 274:1133-1138.

Kraszewski K, Mundigl O, Daniell L, Verderio C, Matteoli M, De Camilli P (1995) Synaptic vesicle dynamics in living cultured hippocampal neurons visualized with CY3-conjugated antibodies directed against the lumenal domain of synaptotagmin. J Neurosci 15:4328-4342.

LeVay S, Wiesel TN, Hubel DH (1980) The development of ocular dominance columns in normal and visually deprived monkeys. J Comp Neurol 191:1-51.

Lichtman JW, Colman H (2000) Synapse elimination and indelible memory. Neuron 25:269-278.

Lissin DV, Gomberts SN, Carroll RC, Christine CW, Kalman D, Kitamura M, Hardy S, Nicoll RA, Malenka RC, von Zastrow M (1998) Activity differentially regulates the surface expression of synaptic AMPA and NMDA glutamate receptors. Proc Natl Acad Sci USA 95:7097-7102.

Ma L, Zablow L, Kandel ER, Siegelbaum SA (1999) Cyclic AMP induces functional presynaptic boutons in hippocampal CA3-CA1 neuronal cultures. Nat Neurosci 2:24-30.

Markram H, Luebke J, Frotscher M, Sakmann B (1997) Regulation of synaptic efficacy by coincidence of postsynaptic APs and EPSPs. Science 275:213-215.

Murthy VN, Sejnowski TJ, Stevens CF (1997) Heterogeneous release properties of visualized individual hippocampal synapses. Neuron 18:599-612.

Nguyen QT, Lichtman JW (1996) Mechanism of synapse disassembly at the developing neuromuscular junction. Curr Opin Neurobiol 6:104-112.

Rao A, Craig AM (1997) Activity regulates the synaptic localization of the NMDA receptor in hippocampal neurons. Neuron 19:801-812.

Ryan TA, Reuter H, Wendland B, Schwiezer FE, Tsien RW, Smith SJ (1993) The kinetics of synaptic vesicle recycling measured at single presynaptic boutons. Neuron 11:713-724.

Ryan TA, Smith SJ (1995) Vesicle pool mobilization during action potential firing at hippocampal synapses. Neuron 14:983-989.

Ryan TA, Ziv NE, Smith SJ (1996) Potentiation of evoked vesicle turnover at individually resolved synaptic boutons. Neuron 17:125-134.

Ryan TA, Reuter H, Smith SJ (1997) Optical detection of a quantal presynaptic membrane turnover. Nature 388:478-482.

Schikorski T, Stevens CF (1997) Quantitative ultrastructural analysis of hippocampal excitatory synapses. J Neurosci 17:5858-5867.

Sporns O, Jenkinson S (1997) Potassium ion- and nitric oxide-induced exocytosis from populations of hippocampal synapses during synaptic maturation in vitro. Neuroscience 80:1057-1073.

Staple JK, Osen-Sand A, Benfenati F, Pich EM, Catsicas S (1997) Molecular and functional diversity at synapses of individual neurons in vitro. Eur J Neurosci 9:721-731.

Stryker MP, Harris WA (1986) Binocular impulse blockade prevents the formation of ocular dominance columns in cat visual cortex. J Neurosci 6:2117-2133.

Valtorta F, Benfenati F, Greengard P (1992) Structure and function of the synapsins. J Biol Chem 267:7195-7198.

Ziv NE, Smith SJ (1996) Evidence for a role of dendritic filopodia in synaptogenesis and spine formation. Neuron 17:91-102. 